\title{
Erratum to: Preparation of macroporous polyHIPE foams via radiation-induced polymerization at room temperature
}

\author{
Donglin Mao $\cdot$ Tingting Li $\cdot$ Huarong Liu $\cdot$ Zichao Li • \\ Hong Shao $\cdot$ Min Li
}

Published online: 16 March 2013

(C) Springer-Verlag Berlin Heidelberg 2013

\section{Erratum to: Colloid Polym Sci}

\section{DOI 10.1007/s00396-013-2899-8}

The original version of this article, unfortunately, contained an error.

Figures 2, 3, and 4 captions were incorrect. The correct ones are as follows:

Fig. 2 Macroporous $\mathrm{P}(\mathrm{St}-\mathrm{DVB})$ foams with different concentration of Span 80 and the internal aqueous phase fraction prepared by radiation-induced polymerization at room temperature. (a) SS1W80r; (b) SS1.4W80r; (c) SS1.4W90r; (d) SS3W80r; (e) SS3W90r. Scale bar= $100 \mu \mathrm{m}$
Fig. 3 Macroporous P(St-DVB) foams with different concentration of Span 80 and the internal aqueous phase fraction polymerized at $60^{\circ} \mathrm{C}$. (a) SS5W80c; (b) SS5W90c; (c) SS10W80c; (d) SS10W90c. Scale bar $=100 \mu \mathrm{m}$

Fig. 4 Macroporous P(MMA-DVB) foams with different concentration of Span 80 and internal aqueous phase fraction prepared by radiation-induced polymerization at room temperature. (a) MS20W90r; (b) MS18W90r; (c) MS20W90rh; (d) MS18W90rh; (e) MS16W90rh; (f) MS20W75r. Scale bar= $10 \mu \mathrm{m}$

The online version of the original article can be found at http://dx.doi.org/ 10.1007/s00396-013-2899-8

D. Mao $\cdot$ T. Li $\cdot$ H. Liu $(\bowtie) \cdot \mathrm{Z}$. Li $\cdot$ H. Shao

CAS Key Laboratory of Soft Matter Chemistry, Department

of Polymer Science and Engineering, University of Science

and Technology of China, Hefei, Anhui 230026,

People's Republic of China

e-mail: hrliu@ustc.edu.cn

M. Li

Zhejiang Test Academy of Quality and Technical Supervision, Hangzhou, Zhejiang 310013, People's Republic of China 